\title{
An Eulerian moving front algorithm with weak-form tip asymptotics for modeling hydraulically driven fractures
}

\author{
Anthony Peirce ${ }^{1, * \dagger}$ and Emmanuel Detournay ${ }^{2,3}$ \\ ${ }^{1}$ Department of Mathematics, University of British Columbia, Vancouver, BC, Canada \\ ${ }^{2}$ University of Minnesota, Minneapolis, MN, U.S.A. \\ ${ }^{3}$ CSIRO Petroleum, Australia
}

\begin{abstract}
SUMMARY
The coupled equations for a propagating hydraulic fracture (HF) exhibit a multi-scale structure for which resolution on all length scales becomes computationally prohibitive. Asymptotic analysis is able to identify the dominant physical process active at the computational length scale. This paper describes a novel algorithm that uses weak-form tip asymptotics on a rectangular Eulerian mesh to solve the problem of a propagating HF. The location of the fracture front is determined within each tip element by matching the volume associated with the known asymptotic solution to the flux of fluid into the given element. Even if the fracture front is curved, the algorithm is able to capture the solution on a relatively coarse rectangular mesh by implementing a weak form of the tip asymptotic solution, based on averaging the volume over a tip element to determine the fracture widths at tip element centers. The fracture is divided into a 'channel' region made up of elements that are filled with fluid and a 'tip' region comprising partially filled elements. An iterative procedure is used to determine the fracture widths and fluid pressures in the channel region and the fracture font locations in the tip regions. The algorithm is tested by analyzing an HF propagating in a viscosity-dominated regime within an impermeable homogeneous elastic material for which a similarity solution is available. The numerical results show close agreement with the exact solution even though a relatively coarse mesh is used. Copyright (C) 2008 John Wiley \& Sons, Ltd.
\end{abstract}

Received 16 August 2007; Revised 4 February 2008; Accepted 5 February 2008

KEY WORDS: moving front; hydraulic fracture; fracture mechanics

\footnotetext{
*Correspondence to: Anthony Peirce, Department of Mathematics, University of British Columbia, 121-1984 Mathematics Road, Vancouver, BC, Canada V6T 1Z2.

${ }^{\dagger}$ E-mail: peirce@math.ubc.ca

Contract/grant sponsor: NSERC; contract/grant number: 195803-02

Contract/grant sponsor: National Science Foundation; contract/grant number: 0600058
}

Copyright (C) 2008 John Wiley \& Sons, Ltd. 


\section{INTRODUCTION}

Hydraulic fractures (HFs) are a class of tensile fractures that propagate in brittle materials by the injection of a pressurized viscous fluid. Natural examples of HF include the formation of dykes $[1-3]$ and sills $[4,5]$ by the intrusion of pressurized magma from deep chambers. HFs are also used in a multiplicity of engineering applications, including the deliberate formation of fracture surfaces in granite quarries [4,6], waste disposal [7,8], remediation of contaminated soils [9-11], cave inducement in mining $[12,13]$, and fracturing of hydrocarbon-bearing rocks in order to enhance the production of oil and gas wells [14-16].

The design of robust numerical algorithms to model HF poses considerable challenges due to the complex structure of the governing equations (see [17] for a recent review paper), comprising a hypersingular integral equation that expresses the balance of fluid pressure within the fracture with the elastic response of the rock mass, a degenerate lubrication equation that governs the flow of viscous fluid within the fracture, and a free boundary problem determined by a fracture propagation condition in which the stress intensity factor along the periphery of the fracture is in limit equilibrium with the local fracture toughness of the material. Recent asymptotic analyses [18-21] of these coupled equations have identified a multi-scale structure in which different physical processes compete. For example, the energy dissipated in driving the viscous fluid through the fracture competes with the energy expended in breaking the rock. The selection of the dominant physical process depends on the time scale referred to as the initiation of the fracture and length scale referred to as the fracture tip. A surprising result of this analysis is that, even though a fracture may be propagating in a medium with a finite toughness, when viewed on the computational length scale (i.e. one element size), the fracture width may not exhibit the square root asymptotic behavior that one would expect from linear elastic fracture mechanics (LEFM). Indeed, resolving the finer, toughness length scale may, in fact, be computationally prohibitive. On the other hand, the ability to identify the dominant physical process at the element length scale also affords us the opportunity to be able to considerably reduce the required computational resources, provided we use the appropriate tip asymptotic behavior.

The objective of this paper is to present a novel algorithm that is able to incorporate the appropriate tip asymptotic behavior on a rectangular Eulerian mesh in a weak form-in this case as an average of the asymptotic behavior over a tip element. The method is a generalization to planar fractures of a prototype algorithm originally developed to model the propagation of plane strain HF [22]. The weak-form approach to the implementation of the tip asymptotics enables us to circumvent the considerable complexity of implementing specialized tip shape functions in the discretization of the elastic integral equation for arbitrarily oriented fracture fronts, which are unknown as the fracture progresses. Given the tip asymptotics, we are able to locate the fracture front within a tip element by matching the volume of fluid corresponding to the tip asymptote to the flux of fluid into the element. This approach not only makes it possible to build the tip asymptotics into the solution but simultaneously provides a method to locate the free boundary.

The choice of a rectangular mesh is motivated by the possible extension of the algorithm to account for the layering of the rock mass because such a mesh can exploit the layer-parallel translational invariance of the Green's function matrix [23-25]. We test the efficacy of this algorithm, which is based on rectangular elements, at capturing fractures with non-rectangular footprints by considering a radially symmetric HF for which a similarity solution exists [26]. The influence 
of elastic layers can be incorporated by merely replacing Green's function for a homogeneous medium by the appropriate one for a layered medium.

In Section 2, we present the governing equations as well as the tip asymptotic expansion for an HF propagating in a regime for which viscous dissipation dominates the energy consumed in breaking the rock. In Section 3, we describe details of the novel algorithm, including the discrete equations and the procedure for locating the front position within a tip element as well the method used to compute the front velocity. In Section 4, we demonstrate the performance of the novel algorithm by direct comparison with a similarity solution for an HF propagating in a viscositydominated regime within a spatially uniform impermeable medium. In Section 5, we make some concluding remarks.

\section{MATHEMATICAL MODEL}

\subsection{Assumptions}

The equations governing the propagation of an HF in a reservoir have to account for the dominant physical processes taking place during the treatment, namely the deformation of the rock, the creation of new fracture surfaces, the flow of the fracturing fluid in the crack, the formation of a cake, and the leak-off of the fracturing fluid into the reservoir. In order to minimize the energy expended, fractures in pre-stressed media typically propagate in a plane normal to the least compressive stress. Besides the standard assumptions regarding the applicability of LEFM and lubrication theory, we make a series of simplifications that can readily be justified for the purposes of this contribution: (i) the rock is homogeneous (having uniform values of toughness $K_{I c}$, Young's modulus $E$, and Poisson's ratio $v$ ), (ii) the fracturing fluid is incompressible and Newtonian (having a viscosity $\mu$ ), (iii) the fracture is always in limit equilibrium, (iv) the rock is impermeable, (v) gravity is neglected in the lubrication equation, and (vi) the fluid front coincides with the crack front, because the lag between the two fronts is negligible under typical high confinement conditions of reservoir stimulation $[18,27]$. Note that the assumptions regarding the homogeneity of $K_{I c}$ and $\mu$ can be relaxed without any significant changes to the model.

As discussed further below, we will restrict our discussion to cases where the fracture is propagating in the viscosity-dominated regime, i.e. under conditions where the solution, at the fracture scale, does not depend on the toughness $K_{I c}$ and is actually virtually the same as the zero toughness solution.

\subsection{Mathematical formulation}

The solution to this problem comprises the fracture aperture $w(x, y, t)$, the fluid pressure $p_{\mathrm{f}}(x, y, t)$, the flux $\mathbf{q}(x, y, t)$, and the position of the front $C(t)$, where $t$ denotes the time and $x, y$ are the coordinates in a system of axes referenced to the injection point (Figure 1). The solution depends on the injection rate $Q(t)$, the far-field compressive stress $\sigma(x, y)$ perpendicular to the fracture plane (a known function of position), and the three material parameters $\mu^{\prime}, E^{\prime}, K^{\prime}$ defined as

$$
\mu^{\prime}=12 \mu, \quad E^{\prime}=\frac{E}{1-v^{2}}, \quad K^{\prime}=4\left(\frac{2}{\pi}\right)^{1 / 2} K_{I c}
$$




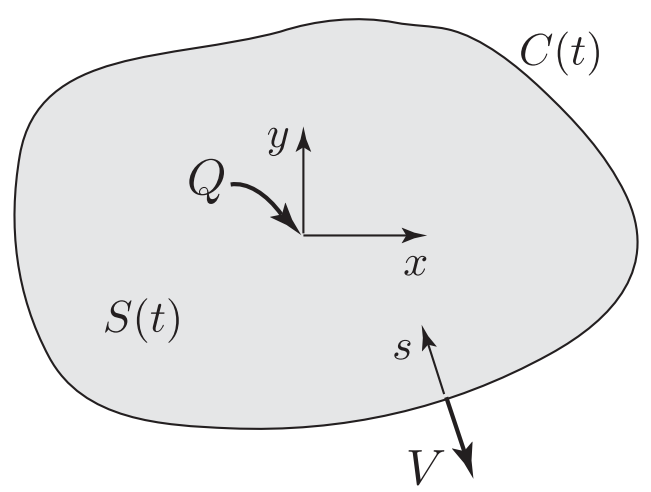

Figure 1. Sketch of a planar fracture.

Here $E^{\prime}$ is the plane strain modulus while the alternate viscosity $\mu^{\prime}$ and toughness $K^{\prime}$ are introduced to keep equations uncluttered by numerical factors. The front $C(t)$ and the field quantities $w(x, y, t)$, $p_{\mathrm{f}}(x, y, t)$, and $\mathbf{q}(x, y, t)$ are governed by a set of equations arising from LEFM, lubrication theory, and the associated boundary conditions.

2.2.1. Elasticity. The equation relating the displacement and stress field in the homogeneous elastic medium can be condensed into a hypersingular integral equation between the fracture aperture $w$ and the fluid pressure $p_{\mathrm{f}}[28,29]$

$$
p_{\mathrm{f}}-\sigma=-\frac{E^{\prime}}{8 \pi} \int_{S(t)} \frac{w\left(x^{\prime}, y^{\prime}, t\right) \mathrm{d} S\left(x^{\prime}, y^{\prime}\right)}{\left[\left(x^{\prime}-x\right)^{2}+\left(y^{\prime}-y\right)^{2}\right]^{3 / 2}}
$$

where $S(t)$ denotes the fracture footprint (enclosed by the crack front $C(t)$ and having a characteristic dimension $L(t))$.

2.2.2. Lubrication. The lubrication equation consists of the Reynolds equation

$$
\frac{\partial w}{\partial t}=\frac{1}{\mu^{\prime}} \nabla \cdot\left(w^{3} \nabla p_{\mathrm{f}}\right)+Q(t) \delta(x, y)
$$

which is obtained by combining Poiseuille's law

$$
\mathbf{q}=-\frac{w^{3}}{\mu^{\prime}} \nabla p_{\mathrm{f}}
$$

and the continuity equation

$$
\frac{\partial w}{\partial t}+\nabla \cdot \mathbf{q}=Q(t) \delta(x, y)
$$

where $\delta(x, y)$ denotes the two-dimensional Dirac function. 
2.2.3. Boundary conditions at the moving front $C(t)$. Assuming that the fracture is always in limit equilibrium at each point along the fracture front, LEFM [30] implies that the fracture aperture in the immediate vicinity of the front is given by

$$
w \sim \frac{K^{\prime}}{E^{\prime}} s^{1 / 2}
$$

where $s$ denotes the distance from the crack front $C(t)$ (with the $s$-axis directed inwards).

The second condition simply expresses a zero-flux boundary condition at the fracture tip

$$
\lim _{s \rightarrow 0} q=\lim _{s \rightarrow 0} w^{3} \frac{\partial p_{\mathrm{f}}}{\partial s}=0
$$

We observe, from the Reynolds equation (3), that because $w \rightarrow 0$ as $s \rightarrow 0$ it follows that the pressure gradient becomes infinite as $s \rightarrow 0$. Unlike a classical Stefan boundary condition at a moving front where the front velocity is given in terms of quantities having a definite limit at the front, the front velocity has to be extracted from an asymptotic analysis of the non-linear system of equations (2)-(7). In the particular case of an impermeable medium, the front velocity $V$ is equal to the average fluid velocity as $s \rightarrow 0$

$$
V=\frac{1}{\mu^{\prime}} \lim _{s \rightarrow 0} w^{2} \frac{\partial p_{\mathrm{f}}}{\partial s}
$$

which shows that $V$ is the limit of an indeterminate form that poses considerable difficulties for existing free boundary evolution algorithms such as front tracking, the volume of fluid method, or the level set method.

2.2.4. Scaled equations. The system of equations (2), (3)-(7) is closed and can, in principle, be solved to determine the evolution of an HF given appropriate initial conditions. To re-scale these equations, we introduce a length scale $L_{*}$, a time scale $T_{*}$, a characteristic fracture aperture $W_{*}$, and a characteristic (net) pressure $P_{*}$ (all yet to be defined). The physical quantities of the problem are thus formally expressed as

$$
x=L_{*} \chi, \quad y=L_{*} \zeta, \quad t=T_{*} \tau, \quad w=W_{*} \Omega, \quad p_{\mathrm{f}}=P_{*} \Pi_{\mathrm{f}}
$$

Furthermore, in order to scale the equations, we introduce the characteristic injection rate $Q_{0}$ and the characteristic stress $\sigma_{0}$ such that

$$
Q=Q_{0} \psi(\tau), \quad \sigma=\sigma_{0} \varphi(\chi, \zeta)
$$

where $\psi(\tau)$ and $\varphi(\chi, \zeta)$ are known functions that we have already chosen to express in terms of the dimensionless time $\tau$ and space variables $\chi$ and $\zeta$.

In the scaled variables, the governing equations transform to the following:

$$
\begin{aligned}
\Pi_{\mathrm{f}}-\Sigma_{0} \varphi(\chi, \zeta) & =-\frac{1}{8 \pi \mathscr{G}_{e}} \int_{\mathscr{S}(\tau)} \frac{\Omega\left(\chi^{\prime}, \zeta^{\prime}, \tau\right) \mathrm{d} \mathscr{S}\left(\chi^{\prime}, \zeta^{\prime}\right)}{\left[\left(\chi^{\prime}-\chi\right)^{2}+\left(\zeta^{\prime}-\zeta\right)^{2}\right]^{3 / 2}} \\
\frac{1}{\mathscr{G}_{v}} \frac{\partial \Omega}{\partial \tau} & =\frac{1}{\mathscr{G}_{m}} \nabla \cdot\left(\Omega^{3} \nabla \Pi_{\mathrm{f}}\right)+\psi(\tau) \delta(\chi, \zeta) \\
\lim _{\xi \rightarrow 0} \frac{\Omega}{\xi^{1 / 2}} & =\mathscr{G}_{k}, \quad \lim _{\xi \rightarrow 0} \Omega^{3} \frac{\partial \Pi_{\mathrm{f}}}{\partial \xi}=0, \quad v=\lim _{\xi \rightarrow 0} \Omega^{2} \frac{\partial \Pi_{\mathrm{f}}}{\partial \xi}
\end{aligned}
$$


where $\xi$ is the scaled distance from the fracture front $\mathscr{C}(t)$. Here the dimensionless groups $\mathscr{G}$ are defined as follows:

$$
\mathscr{G}_{e}=\frac{L_{*} P_{*}}{E^{\prime} W_{*}}, \quad \mathscr{G}_{k}=\frac{K^{\prime} L_{*}^{1 / 2}}{E^{\prime} W_{*}}, \quad \mathscr{G}_{m}=\frac{\mu^{\prime} Q_{0}}{P_{*} W_{*}^{3}}, \quad \mathscr{G}_{v}=\frac{Q_{0} T_{*}}{L_{*}^{2} W_{*}}
$$

and $\Sigma_{0}$ is the scaled far-field stress $\sigma_{0} / P_{*}$ and the scaled tip velocity is given by $v=V / V_{*}$, where $V_{*}=W_{*}^{2} P_{*} / \mu^{\prime} L_{*}$. Note that it is advantageous to introduce the net pressure $\Pi=\Pi_{\mathrm{f}}-\Sigma_{0}$ if $\varphi(\chi, \zeta)=1$, i.e. if the far-field stress is homogeneous. The characteristic dimension of the fracture (e.g. the fracture radius) is $\gamma(\tau)=L / L_{*}$. By constraining the four dimensionless groups $\mathscr{G}$ in (14) to be equal to 1 , we obtain four conditions that identify the scales $L_{*}, T_{*}, P_{*}$, and $W_{*}$.

2.2.5. Tip asymptotic behavior. If the solid has zero toughness and if the lag between the fluid front and the fracture edge is negligible compared with the fracture characteristic dimension, then the coupling between the elasticity and lubrication equations yields the following tip asymptotic behavior [31]:

$$
\Omega \sim \beta_{m 0} v^{1 / 3} \xi^{2 / 3}
$$

This asymptote also dominates at the fracture scale, even though the solid has a finite toughness, provided that the propagation of the fracture takes place in the viscosity-dominated regime. Indeed, it can be inferred, from the generalized tip asymptote for an HF advancing in a solid with finite toughness [18] and from the thickness $\ell_{c}$ of the asymptotic region, which is of order $O\left(10^{-1} \gamma\right)$, that the $\frac{2}{3}$ asymptote (15) applies if $\ell_{c} \gtrsim v^{-2}$. For a penny-shaped fracture, dissipation associated with viscous flow dominates the fracture growth when $\tau \lesssim 1$ [26]. Under these conditions of 'small' toughness, a boundary layer develops with the classical square root singularity taking place over a length scale of order $O\left(10^{-5} v^{-2}\right)$. The thickness of this boundary layer cannot typically be resolved at the discretization level used in numerical simulations.

\section{PRINCIPLE OF THE NUMERICAL ALGORITHM}

We consider a uniform rectangular mesh with spacings $\Delta \chi$ and $\Delta \zeta$ in the two coordinate directions to encompass the region into which the fracture will move. The fracture surface $\mathscr{S}$ is therefore covered by rectangular elements $\Delta \mathscr{S}_{m, n}$ such that $\mathscr{S} \subset \cup \Delta \mathscr{S}_{m, n}$.

We now discuss the principles behind the multi-scale fixed grid algorithm implemented in the planar HF code MALIKA. The algorithm uses constant displacement discontinuity (DD) elements for the elasticity computations [28] and a five-node finite difference stencil for the fluid-flow calculations [25]. The computational scheme further relies on dividing the fracture into two regions, the 'channel region' comprising those elements filled with fluid, and the 'tip region' that is under the asymptotic umbrella and comprises the partially filled elements. Tip elements exchange fluid only with their neighboring channel elements and not with other tip elements. The solution comprising the fracture width and the location of the fracture front is achieved at each time step by iterating alternately between the channel elements and the tip elements.

Assuming a known trial solution for the tip elements, the non-linear channel equations are solved to yield the channel element widths. Once an equilibrium solution has been obtained for the 
channel elements, the widths of the channel elements are frozen and the tip solution is adjusted, in the following way, to match the overall volume balance between the two regions. On the boundary between the channel region and the tip region, the fluxes are evaluated to determine the volume of fluid in each tip element. These tip fluid volumes are then used to locate the fracture front by exploiting the property that, for a smooth front, the asymptotic behavior of the solution close to the tip corresponds to a one-dimensional fracture propagating in a state of plane strain [32]. For example, if the applicable asymptotic power law is of the form $\Omega(\hat{\xi}) \sim c \hat{\xi}^{\alpha}$, then the tip volume $V(\ell)$ can be expressed in the form

$$
V(\ell)=c \int_{0}^{\ell} \hat{\xi}^{\alpha} \phi(\hat{\xi}) \mathrm{d} \hat{\xi}
$$

where $\hat{\xi}$ is the distance from the local fracture front, $\phi(\hat{\xi})$ is the local dimension of the current element in a direction parallel to the front, and $\ell$ is the distance between the front and the opposite vertex of the tip element. From (15) we observe that the asymptotic behavior depends on the tip velocity. Thus, a critical issue for the proposed scheme is the accurate calculation of the local tip velocity, which is extrapolated from the fluid velocity at the channel/tip interface. Average width values, calculated from the tip fluid volumes, are then allocated to the tip width values in order to set the current tip trial solution. We then freeze the tip solution and return to the solution of the non-linear channel equations described above. The staggered computational scheme involves a two-way communication between the channel and tip calculations. That is, a new estimate of the flux (magnitude and direction) is provided to the tip at the end of a channel calculation, while the tip computation returns an updated aperture for the tip elements, which affects the channel solution via the non-local elasticity operator. This process is repeated until both the front position and the channel and tip solutions reach equilibrium.

\subsection{Discrete equations}

3.1.1. Elasticity: constant strength DDs. The elasticity equation (11) is discretized by assuming that the fracture opening $\Omega(\chi, \zeta, \tau)$ is piecewise constant over each rectangular element $\Delta \mathscr{S}_{m, n}$, i.e.

$$
\Omega(\chi, \zeta, \tau)=\sum_{m, n} \Omega_{m, n}(\tau) H_{m, n}(\chi, \zeta)
$$

in which

$$
H_{m, n}(\chi, \zeta)= \begin{cases}1 & \text { for }(\chi, \zeta) \in \Delta \mathscr{S}_{m, n} \\ 0 & \text { for }(\chi, \zeta) \notin \Delta \mathscr{S}_{m, n}\end{cases}
$$

is the characteristic function for element $(m, n)$. Substituting this approximation into the integral equation (11) and evaluating the pressures at the collocation points comprising the element centers yield a system of algebraic equations of the form

$$
\Pi_{k, l}(\tau)=\sum_{m, n} C_{k-m, l-n} \Omega_{m, n}(\tau)
$$


where

$$
C_{k-m, l-n}=-\frac{1}{8 \pi}\left[\frac{\sqrt{\left(\chi_{k}-\chi\right)^{2}+\left(\zeta_{l}-\zeta\right)^{2}}}{\left(\chi_{k}-\chi\right)\left(\zeta_{l}-\zeta\right)}\right]_{\chi=\chi_{m}-\Delta \chi / 2, \zeta=\zeta_{n}-\Delta \zeta / 2}^{\chi=\chi_{m}+\Delta \chi / 2, \zeta=\zeta_{n}+\Delta \zeta / 2}
$$

3.1.2. Lubrication: discretized Reynolds equation. In order to discretize the fluid-flow equation (12), we integrate first with respect to time over the interval $[\tau-\Delta \tau, \tau]$ and, to achieve a backward Euler time-marching scheme, we approximate the time integral of the pressure gradient term by the endpoint rule. We then integrate over a spatial element $\Delta \mathscr{S}_{e}$ and apply the divergence theorem to obtain

$$
\int_{\Delta \mathscr{S}_{e}} \Omega(\tau)-\Omega(\tau-\Delta \tau) \mathrm{d} \mathscr{S}=\Delta \tau\left[\int_{\Delta \mathscr{C}_{e}} \Omega^{3} \frac{\partial \Pi}{\partial n} \mathrm{~d} \mathscr{C}\right]_{\tau}+\delta_{m, 0} \delta_{n, 0} \int_{\tau-\Delta \tau}^{\tau} \psi\left(\tau^{\prime}\right) \mathrm{d} \tau^{\prime}
$$

where $\delta_{i, j}$ is the Kronecker delta symbol. In the above, we have assumed that the injection point is located at the center of element $\Delta \mathscr{S}_{0,0}$. We use pressures $\Pi_{k, l}(\tau)$ and average widths $\Omega_{k, l}(\tau)$ sampled at element centers along with central difference approximations to the pressure gradients on the boundaries of the elements and divide by $\Delta \chi \Delta \zeta$ to obtain

$$
\Omega_{i, j}(\tau)-\Omega_{i, j}(\tau-\Delta \tau)=\Delta \tau[A(\Omega) \Pi]_{i, j}+\frac{\delta_{m, 0} \delta_{n, 0}}{\Delta \chi \Delta \zeta} \int_{\tau-\Delta \tau}^{\tau} \psi\left(\tau^{\prime}\right) \mathrm{d} \tau^{\prime}
$$

where $A(\Omega)$ is the difference operator defined by

$$
\begin{aligned}
{[A(\Omega) \Pi]_{k, l}=} & \frac{1}{\Delta \chi}\left(\Omega_{k+1 / 2, l}^{3} \frac{\left(\Pi_{k+1, l}-\Pi_{k, l}\right)}{\Delta \chi}-\Omega_{k-1 / 2, l}^{3} \frac{\left(\Pi_{k, l}-\Pi_{k-1, l}\right)}{\Delta \chi}\right) \\
& +\frac{1}{\Delta \zeta}\left(\Omega_{k, l+1 / 2}^{3} \frac{\left(\Pi_{k, l+1}-\Pi_{k, l}\right)}{\Delta \zeta}-\Omega_{k, l-1 / 2}^{3} \frac{\left(\Pi_{k, l}-\Pi_{k, l-1}\right)}{\Delta \zeta}\right)
\end{aligned}
$$

\subsection{Discrete equations for the tip}

3.2.1. Front position and front velocity. The tip calculation involves computing the front position within each tip element and the front velocity vector. During the iterations for a new time step, we use the front speed $v_{0 k}$ and orientation $\alpha_{0 k}, k=1: n_{t}$, that are computed at the end of the previous time step, in order to compute the solution comprising the channel width $\Omega_{i, j}$, the tip width $\Omega_{k}$, and the front position. Because the orientation of the front is assumed to be perpendicular to the frozen front velocity vector $\mathbf{v}_{0 k}$, calculation of the front position in a tip element $k$ is reduced to determining the distance $\ell_{k}$ between the front segment and the point of first entry of the front into the tip element (which is generally a corner, but could also be an edge of the tip element), see Figure 2. Note that the point of first entry could possibly change between time steps, due to variation in the estimated orientation $\alpha_{k}$ of the front velocity. Once the iterative scheme for computing the solution for the current time step has converged, a new estimate of the front velocity $\mathbf{v}_{k}$ can be assessed in each tip element, and the algorithm can then proceed to a new time step.

Calculating the front position ensures that the fluid mass is conserved, i.e. the volume of fluid $\mathscr{V}_{k}$ that has accumulated in tip element $k$ through fluid exchange between this element and the adjacent 


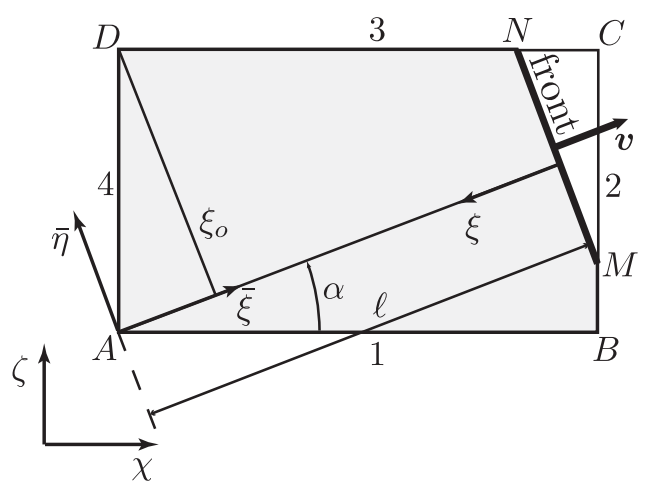

Figure 2. Tip element.

channel elements (which is calculated in the channel computations) is allocated to $\Omega_{k} \Delta \chi \Delta \zeta$, where $\Omega_{k}$ is the average DD $\Omega_{i, j}$ at the center of the $i, j$ th element. If the volume influx into a given element exceeds that which is consistent with the asymptotic behavior, then that element is moved from the tip set to the channel and its north, south, east, or west neighbors, which were hitherto dry are then activated as tip elements. On the other hand, the front velocity $\mathbf{v}_{0 k}$ is estimated from an interpolated flux field by combining information from adjacent elements (both tip and channel) without explicitly enforcing volume balance. The front velocity will affect the front location but not the conservation of fluid volume. Indeed, it is possible to find numerous slightly different solutions that both equilibrate forces and conserve fluid volume, but which are each associated with slightly different fracture footprints. Identifying the correct footprint is thus the challenge for the front location algorithm.

Calculating the front position $\ell$ (at each iteration) and the front velocity $\mathbf{v}$ (once the iterations have converged) in a tip element is based on the autonomous tip solution discussed earlier. In other words, the critical feature of the algorithm rests on the assumption that the tip element is entirely within the asymptotic umbrella. The other assumptions are that the front velocity does not change while it is traversing an element and that the front is straight (a corollary of the assumption of having only one value of the front velocity in the element). Adopting the asymptotic solution in the tip implies that the local flux field $\boldsymbol{\Psi}$ and the aperture $\Omega$, which will be used to evaluate the position and velocity of the front, only depend on the distance $\xi$ from the front and on the front speed $v$; and also that the flux $\boldsymbol{\Psi}$ is assumed to point in the same direction as $\mathbf{v}$.

3.2.2. Tip volume balance equation. The fluid volume injected into a tip element is given by the line integral in (20) involving the normal flux vectors $\Psi_{n}=-\Omega^{3} \partial \Pi / \partial n$ along the faces of a tip element. Assuming that the injection source does not fall in a tip element and using the same finite difference scheme as for the channel calculations the discrete lubrication equation for a tip element assumes the form

$$
\Delta \mathscr{V}_{k}^{(l)}=\mathscr{V}_{k}^{(l)}-\mathscr{V}_{0, k}^{(l)}=\Delta t \sum_{i=1}^{4} \Psi_{k, i}^{(l)} \Delta s_{i}
$$


where $\mathscr{V}_{k}^{(l)}=\int_{\Delta \mathscr{S}_{e}} \Omega(\tau) \mathrm{d} \mathscr{S}, \mathscr{V}_{0, k}^{(l)}=\int_{\Delta \mathscr{S}_{e}} \Omega(\tau-\Delta \tau) \mathrm{d} \mathscr{S}$, and $\Psi_{k, i}^{(l)}$ is the average normal flux across edge $i$ of tip element $k$, which is non-zero only if the edge $i$ is shared by a tip and a channel element. See Figure 2 for the edge numbering convention. Note that $\Delta s_{i}=\Delta \chi$ for $i=1,3$ and $\Delta s_{i}=\Delta \zeta$ for $i=2,4$.

3.2.3. Calculating the front position. The current front position $\ell$ can be calculated from the tip volume balance relation (23), which can be re-written in the following form:

$$
\mathscr{V}(\ell)=\mathscr{V}_{0}+\Delta \mathscr{V}
$$

To determine an expression for $\mathscr{V}(\ell)$ corresponding to an asymptotic relation of the form (15), it is convenient to define the power law function $\Xi_{a}(\xi)$ as

$$
\Xi_{a}=\xi^{a}, \quad a>-1
$$

which enables us to express the volume stored in a tip element as

$$
\mathscr{V}(\ell)=\frac{9}{40} \beta_{m o} v^{1 / 3} \breve{\Xi}_{8 / 3}(\ell)
$$

The function $\breve{F}(\ell)$ denotes the tip integral operator defined as [32]

$$
I(\ell)=\int_{\Delta \mathscr{S}_{\ell}} \frac{\mathrm{d} f}{\mathrm{~d} \xi} \mathrm{d} \mathscr{S}
$$

where the function $f(\xi)$ vanishes at $\xi=0$ and $\Delta \mathscr{S}_{\ell}$ is the fluid-filled part of a tip element (represented by the gray fill in Figure 2). The integral $\breve{F}(\ell)$ can be solved in closed form as

$$
\breve{F}(\ell)=\left\{\begin{array}{l}
m F(\ell), \quad 0 \leqslant \ell \leqslant \xi_{0}, \quad m \neq \infty \\
m\left[F(\ell)-F\left(\ell-\xi_{0}\right)\right], \quad \xi_{0} \leqslant \ell \leqslant \lambda-\xi_{0}, \quad m \neq \infty \\
m\left[F(\ell)-F\left(\ell-\xi_{0}\right)-F\left(\ell+\xi_{0}-\lambda\right)\right], \quad \lambda-\xi_{0} \leqslant \ell \leqslant \lambda, \quad m \neq \infty \\
\Delta \xi f(\ell), \quad m=\infty
\end{array}\right.
$$

with $\lambda$ representing the maximum value of $\ell$

$$
\lambda=\Delta \chi \cos \alpha+\Delta \zeta \sin \alpha
$$

The shape/geometry of the flow domain depends not only on $\ell$ and $\lambda$ but also on the factor $m$ defined as

$$
m=\frac{1}{\cos \alpha \sin \alpha}
$$

and on the length $\xi_{0}$ given by (see Figure 2)

$$
\xi_{0}= \begin{cases}\Delta \zeta \sin \alpha, & 0 \leqslant \tan \alpha \leqslant \Delta \chi / \Delta \zeta \\ \Delta \chi \cos \alpha, & \Delta \chi / \Delta \zeta \leqslant \tan \alpha<\infty\end{cases}
$$


Indeed, four different flow configurations arise depending on $\ell, \lambda, \xi_{0}$, and $m$. If $m=\infty$, the front is parallel to one of the fixed coordinate axes $(\chi$ or $\zeta)$ and $\Delta \mathscr{S}_{\ell}$ is a rectangle. If $m \neq \infty, \Delta \mathscr{S}_{\ell}$ is either a triangle, quadrilateral, or a pentagon depending on whether $0<\ell<\xi_{0}$, or $\xi_{0}<\ell<\lambda-\xi_{0}$, or $\lambda-\xi_{0}<\ell<\lambda$, respectively. The geometry of $\Delta \mathscr{S}_{\ell}$ affects the evaluation of the surface integral that arises in the calculation of $\ell$.

Assuming that the solution in the channel elements is known, so that the right-hand side of (24) is prescribed, this equation thus becomes a non-linear equation for the length $\ell$.

3.2.4. Computing the front velocity. At the end of the time step, when the solution has converged (i.e. the opening and the front position have been determined), we need to update the front velocity in each tip element, i.e. both its magnitude $v$ and its orientation $\alpha$. We consider the calculation of $v$ and $\alpha$ separately. The calculations of $v$ and $\alpha$ rely in part on a certain average flux in the tip element, which is obtained by averaging the flux over the tip element and its neighbors (both tip and channel elements).

First consider the computation of the average flux $\langle\boldsymbol{\Psi}\rangle$ in a channel element $(i, j)$. The finite difference operator leads to the determination of the normal flux $\Psi_{i, j ; l}$ on each side $l$ of the element $(i, j)$. Hence,

$$
\left\langle\Psi_{\chi}\right\rangle_{i, j}=\Psi_{i, j ; 2}-\Psi_{i, j ; 4}, \quad\left\langle\Psi_{\zeta}\right\rangle_{i, j}=\Psi_{i, j ; 3}-\Psi_{i, j ; 1}
$$

In a tip element, the calculation of $\langle\Psi\rangle$ is based on the fact that $\Psi_{\bar{\eta}}=0$ in the element. Thus,

$$
\left\langle\Psi_{\bar{\xi}}\right\rangle=\frac{1}{\left\|\Delta \mathscr{S}_{\ell}\right\|} \int_{\Delta \mathscr{S}_{\ell}} \Psi_{\bar{\xi}} \mathrm{d} \mathscr{S}_{\ell}, \quad\left\langle\Psi_{\bar{\eta}}\right\rangle=0
$$

where $\left\|\Delta \mathscr{S}_{\ell}\right\|$ denotes the area of $\Delta \mathscr{S}_{\ell}$-the fluid-filled part of a tip element. Using (13), the tip velocity $v_{0}$ can then be estimated from

$$
v_{0}=\frac{\left\langle\Psi_{\bar{\xi}}\right\rangle}{\langle\Omega\rangle}
$$

where $\langle\Omega\rangle$ is the average opening of a tip element

$$
\langle\Omega\rangle=\frac{\mathscr{V}}{\left\|\Delta \mathscr{S}_{\ell}\right\|}=\frac{\mathscr{V}}{\varphi \Delta \chi \Delta \zeta}
$$

with $\varphi(\ell)(0<\varphi<1)$ denoting the surface-filling fraction of a tip element defined by $\varphi(\ell)=$ $\left\|\Delta \mathscr{S}_{\ell}\right\| / \Delta \chi \Delta \zeta$. Hence, in a tip element $k$, the Cartesian components of the average flux $\langle\boldsymbol{\Psi}\rangle$ are given by

$$
\left\langle\Psi_{\chi}\right\rangle_{k}=\left\langle\Psi_{\bar{\xi}}\right\rangle_{k} \cos \alpha_{0 k}, \quad\left\langle\Psi_{\zeta}\right\rangle_{k}=\left\langle\Psi_{\bar{\xi}}\right\rangle_{k} \sin \alpha_{0 k}
$$

Updating the orientation of the front velocity $\alpha$ is based on an interpolated flux $[\Psi]_{i, j}$ in a tip element $(i, j)$ defined as

$$
[\Psi]_{i, j}=\frac{\bar{\Sigma}_{i, j} \varphi_{i, j}\langle\boldsymbol{\Psi}\rangle_{i, j}}{\bar{\Sigma}_{i, j} \varphi_{i, j}}
$$


where the summation $\bar{\Sigma}_{i, j} g_{i, j}$ sums the nodal quantity $g_{i, j}$ over a five-noded stencil centered on $(i, j)$, i.e.

$$
\bar{\Sigma}_{i, j} g_{i, j}=g_{i, j}+g_{i-1, j}+g_{i+1, j}+g_{i, j-1}+g_{i, j+1}
$$

Hence,

$$
\alpha_{i, j}=\arctan \frac{\left[\Psi_{\zeta}\right]_{i, j}}{\left[\Psi_{\eta}\right]_{i, j}}
$$

Naturally, this averaging procedure might involve a nine-noded rather than a five-noded stencil.

\section{NUMERICAL RESULTS}

In this section we consider, as a test case, a fracture propagating in an impermeable homogeneous elastic material in the viscosity-dominated regime. This mode of HF propagation has been shown to be generic for prevailing conditions in treatments of deep low permeability underground reservoirs [33]. We compare the numerical results generated by MALIKA with the corresponding radial solution [26]. For the example considered, we used square elements with $\Delta \chi=\Delta \zeta=1$, a time step $\Delta \tau=4.42$ units, and choose $\psi(\tau)=1$.

In Figure 3, we superimpose fracture fronts at times $\tau=[22.1,128.1,238.6,459.4,901.2,1343.0]$ on the computational grid used by MALIKA. The fronts corresponding to the exact solution are indicated by the solid curves, while the points of intersection of the MALIKA front segments with the computational mesh are indicated by open circles and are connected by dashed lines. The numerical and exact fronts show close agreement given the coarseness of the computational mesh. In Figure 4, we plot cross sections of the width surface $\Omega(\chi, \zeta, \tau)$ with the plane $\zeta=0$

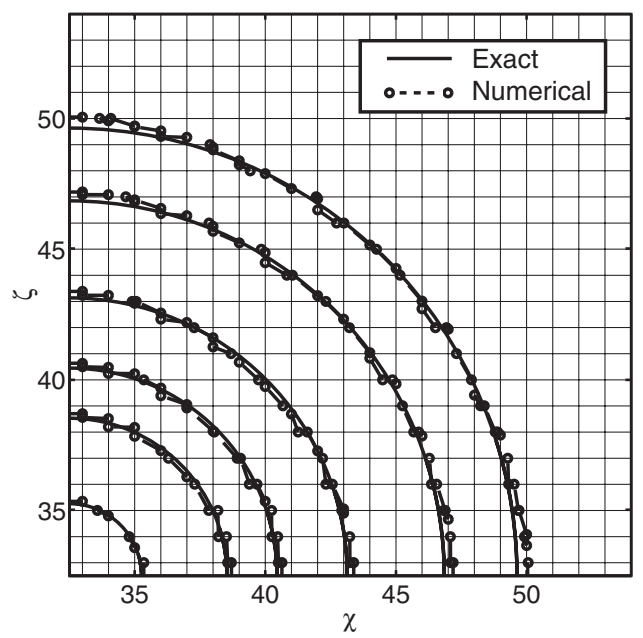

Figure 3. First-quadrant intersection with the fracture footprints that correspond to times $\tau=[22.1,128.1,238.6,459.4,901.2,1343.0]$ and comparison with the $M$-vertex solution. 


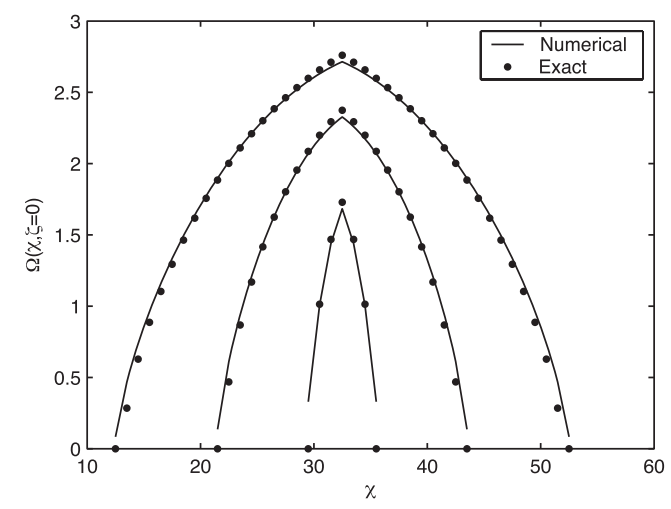

Figure 4. Comparison between the cross sections of the width surface $\Omega(\chi, \zeta, \tau)$ with the plane $\zeta=0$ corresponding to the times $\tau=[26.5,459.4,1784.7]$ obtained numerically (solid lines) and from the exact solution (solid circles).

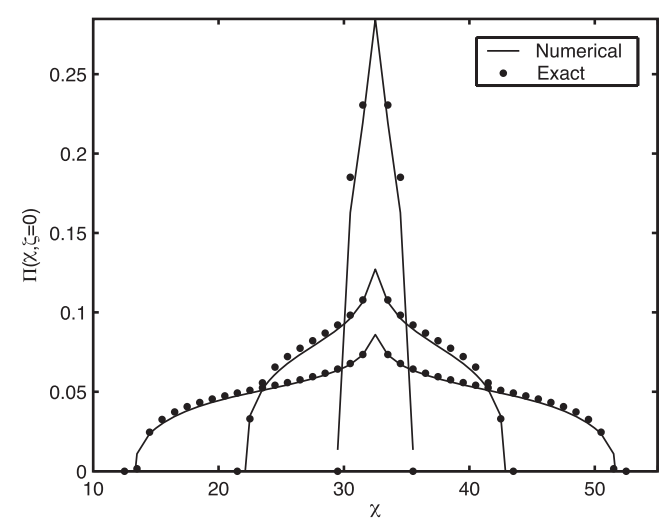

Figure 5. Comparison between the cross sections of the net pressure surface $\Pi(\chi, \zeta, \tau)$ with the plane $\zeta=0$ corresponding to the times $\tau=[26.5,459.4,1784.7]$ obtained numerically (solid lines) and from the exact solution (solid circles).

corresponding to the times $\tau=[26.5,459.4,1784.7]$. The numerical solutions (solid lines) show close agreement with the corresponding exact solutions (solid circles) even for these relatively coarse meshes. We observe that the accuracy of the numerical solutions improve with time as more elements are used to represent the solution. In Figure 5, we plot a sequence of cross sections of the pressure surface $\Pi(\chi, \zeta, \tau)$ with the plane $\zeta=0$ corresponding to the times $\tau=[26.5,459.4,1784.7]$. The numerical pressures (solid lines) show close agreement with the corresponding exact pressures (solid circles) given the coarseness of the mesh. As with the widths, the numerical pressures improve with time as more mesh points are used to represent the solution. In Figure 6, we compare the exact fracture radius $\gamma(\tau)$ with the numerical fracture radius computed by averaging the distance between the well bore and interception points between the computational grid lines and the approximate 


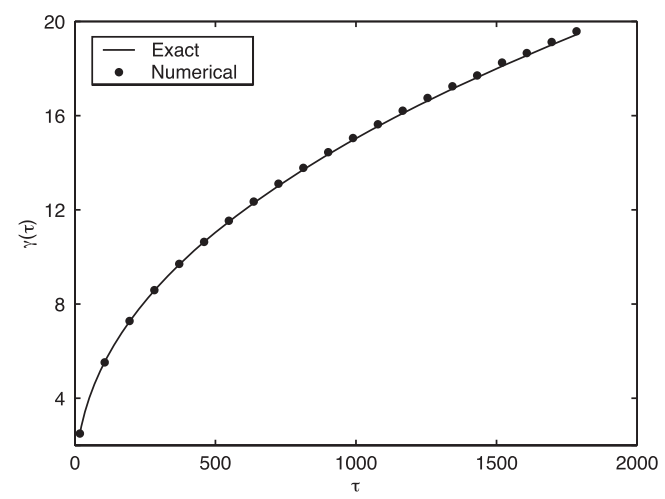

Figure 6. Comparison between the time evolution of the analytic fracture radius $\gamma(\tau)$ and the average numerical fracture radius. The latter is computed by averaging, over the whole perimeter of the fracture, the distance between the well bore and intercepts of the approximate front segments and the computational grid lines.

front segments over the whole perimeter of the fracture, i.e. those points denoted by open circles in Figure 3. There is close agreement between these two solutions.

\section{CONCLUSIONS}

In this paper, we have presented a novel algorithm to solve the governing equations for a propagating HF. These equations pose a number of challenges for the design of robust numerical solution algorithms, including the hypersingular elastic integral equation, the non-linear Reynolds lubrication partial differential equation, and the free boundary problem for the fracture front. These coupled equations exhibit a multi-scale structure for which resolution on all scales would exceed the available computational resources. However, recent asymptotic analyses have been able to identify the appropriate physical process that is dominant at the computational length scale, while those at finer length scales may be considered to be sub-dominant to the fracture propagation process. This observation opens the possibility of achieving relatively accurate numerical approximation schemes, provided that the fracture propagation is determined by the dominant process active at the computational length scale and that the appropriate asymptotic behavior has been incorporated.

To allow for easy extension to layered elastic materials, we have assumed a rectangular Eulerian mesh for the discretization of the governing equations. The proposed algorithm notionally divides the active elements representing a given fracture into two sets- the channel elements that are filled with fluid and the tip elements that are partially filled with fluid. The novel algorithm iterates between the channel and tip sets in order to locate the fracture front and to determine the unknown field variables comprising the fracture width and fluid pressure. Assuming that the solution in the set of channel elements is known, the flux of fluid between the channel and tip elements can be determined. Once the volume of fluid that flows into a tip element is known, then, given that the solution in the tip is approximated by the identified asymptotic behavior, it is possible to locate the unknown fracture front within a tip element. The volume of fluid in each tip element is then 
averaged over the rectangular element to assign a tip width at the element center. Once the tip widths have been determined and the front positions within the tip elements have been located, the tip solution is considered fixed, and the widths in the channel elements are determined by solving the non-local and non-linear system of governing equations. Once the channel widths are known, the pressures throughout the fracture can be determined directly by applying the elasticity operator. Once this step is complete, the channel solution is once again frozen and the tip iteration procedure is re-initiated. These two distinct channel and tip iterative procedures are repeated until the fracture front location process has converged. Once the front location process has converged, the time is advanced, which allows more fluid into the fracture, and the front location process described above is repeated. An alternative algorithm to locate the front is presented in [32].

The novel algorithm described in this paper is tested against a similarity solution for a radial fracture propagating in an impermeable homogeneous elastic medium under a viscosity-dominated regime (i.e. in which the toughness behavior is at a much smaller length scale than that of a computational element). The numerical solution generated by the novel algorithm shows close agreement with the front positions and field variables given by the exact solution in spite of the fact that a relatively coarse mesh is used.

\section{ACKNOWLEDGEMENTS}

A. Peirce gratefully acknowledges the support of the NSERC discovery grants program and the MTS visiting Professorship in Geomechanics for sponsoring a two-week visit to the University of Minnesota during which time this research project was initiated. E. Detournay acknowledges partial support from the National Science Foundation under Grant No. 0600058. Any opinions, findings, conclusions, or recommendations expressed in this material are those of the authors and do not necessarily reflect the views of the National Science Foundation.

\section{REFERENCES}

1. Spence DA, Turcotte DL. Magma-driven propagation crack. Journal of Geophysical Research 1985; 90:575-580.

2. Lister JR, Kerr RC. Fluid-mechanical models of crack propagation and their application to magma transport in dykes. Journal of Geophysical Research 1991; 10049-10077.

3. Rubin AM. Propagation of magma-filled cracks. Annual Review of Earth and Planetary Science 1995; 23:287-336.

4. Pollard DD, Hozlhausen G. On the mechanical interaction between a fluid-filled fracture and the earth's surface. Tectonophysics 1979; 53:27-57.

5. Fialko Y. On origin of near-axis volcanism and faulting at fast spreading mid-ocean ridges. Earth and Planetary Science Letters 2001; 190:31-39.

6. Watson TL. Granites of the southeastern Atlantic states. U.S. Geological Survey Bulletin 1910; 426:282.

7. Abou-Sayed AS, Andrews DE, Buhidma IM. Evaluation of oily waste injection below the permafrost in Prudhoe Bay field. Proceedings of the California Regional Meetings, Bakersfield, CA. Society of Petroleum Engineers: Richardson, TX, 1989; 129-142.

8. Abou-Sayed AS. Safe injection pressures for disposing of liquid wastes: a case study for deep well injection (SPE/ISRM-28236). Proceedings of the Second SPE/ISRM Rock Mechanics in Petroleum Engineering. Balkema: Rotterdam, 1994; 769-776.

9. Frank U, Barkley N. Remediation of low permeability subsurface formations by fracturing enhancements of soil vapor extraction. Journal of Hazardous Materials 2005; 40:191-201.

10. Murdoch LC. Mechanical analysis of idealized shallow hydraulic fracture. Journal of Geotechnical and Geoenvironmental Engineering 2002; 128(6):488-495.

11. Murdoch LC, Slack WW. Forms of hydraulic fractures in shallow fine-grained formations. Journal of Geotechnical and Geoenvironmental Engineering 2002; 128(6):479-487.

12. Jeffrey RG, Mills KW. Hydraulic fracturing applied to inducing longwall coal mine goaf falls. Pacific Rocks 2000. Balkema: Rotterdam, 2000; 423-430. 
13. van As A, Jeffrey RG. Caving induced by hydraulic fracturing at Northparkes mines. Pacific Rocks 2000. Balkema: Rotterdam, 2000; 353-360.

14. Gidley JL, Holditch SA, Nierode DE, Veatch RW. Recent Advances in Hydraulic Fracturing. SPE Monograph Series, vol. 12. Society of Petroleum Engineers: Richardson, TX, 1989.

15. Valkó P, Economides MJ. Hydraulic Fracture Mechanics. Wiley: Chichester, U.K., 1995.

16. Economides MJ, Nolte KG (eds). Reservoir Stimulation (3rd edn). Wiley: Chichester, U.K., 2000.

17. Adachi J, Siebrits E, Peirce AP, Desroches J. Computer simulation of hydraulic fractures. International Journal of Rock Mechanics and Mining Sciences 2007; 44(5):739-757.

18. Garagash DI, Detournay E. The tip region of a fluid-driven fracture in an elastic medium. Journal of Applied Mechanics (ASME) 2000; 67:183-192.

19. Detournay E, Adachi JI, Garagash DI. Asymptotic and intermediate asymptotic behavior near the tip of a fluid-driven fracture propagating in a permeable elastic medium. In Structural Integrity and Fracture, Dyskin AV, Hu X, Sahouryeh E (eds). Swets \& Zeitlinger: Lisse, The Netherlands, 2002; 9-18.

20. Garagash DI, Detournay E. Plane-strain propagation of a fluid-driven fracture: small toughness solution. Journal of Applied Mechanics (ASME) 2005; 72:916-928.

21. Mitchell SL, Kuske R, Peirce AP. An asymptotic framework for the analysis of hydraulic fractures: the impermeable case. Journal of Applied Mechanics (ASME) 2007; 74(2):365-372.

22. Detournay E, Kresse O. A fixed grid algorithm with weak form tip asymptotics for plane strain hydraulic fractures. Technical Report, University of Minnesota, 2005.

23. Peirce AP, Siebrits E. The scaled flexibility matrix method for the efficient solution of boundary value problems in 2D and 3D layered elastic media. Computer Methods in Applied Mechanics and Engineering 2001; 190: 5935-5956.

24. Peirce AP, Siebrits E. Uniform asymptotic approximations for accurate modeling of cracks in layered elastic media. International Journal of Fracture 2001; 110:205-239.

25. Siebrits E, Peirce AP. An efficient multi-layer planar 3D fracture growth algorithm using a fixed mesh approach. International Journal for Numerical Methods in Engineering 2002; 53:691-717.

26. Savitski AA, Detournay E. Propagation of a fluid-driven penny-shaped fracture in an impermeable rock: asymptotic solutions. International Journal of Solids and Structures 2002; 39(26):6311-6337.

27. Bunger AP, Detournay E. Early time solution for a penny-shaped hydraulic fracture. Journal of Engineering Mechanics (ASCE) 2007; 133(5):534-540.

28. Crouch SL, Starfield AM. Boundary Element Methods in Solid Mechanics. George Allen and Unwin: London, 1983.

29. Hills DA, Kelly PA, Dai DN, Korsunsky AM. Solution of Crack Problems. The Distributed Dislocation Technique. Solid Mechanics and its Applications, vol. 44. Kluwer Academic Publishers: Dordrecht, 1996.

30. Rice JR. Mathematical analysis in the mechanics of fracture. In Fracture, an Advanced Treatise, vol. II, Chapter 3 , Liebowitz H (ed.). Academic Press: New York, NY, 1968; 191-311.

31. Desroches J, Detournay E, Lenoach B, Papanastasiou P, Pearson JRA, Thiercelin M, Cheng AH-D. The crack tip region in hydraulic fracturing. Proceedings of the Royal Society of London, Series A 1994; 447:39-48.

32. Peirce AP, Detournay E. An implicit level set method for modeling hydraulically driven fractures. Computer Methods in Applied Mechanics and Engineering 2008; DOI: 10.1016/j.cma.2008.01.013.

33. Bunger AP, Detournay E, Garagash DI, Peirce AP. Numerical simulation of hydraulic fracturing in the viscositydominated regime. Proceedings of the 2007 SPE Hydraulic Fracturing Technology Conference, College Station, TX, U.S.A., 2007; SPE 110115. 